\title{
Coevolution of Role-Based Cooperation in Multi-Agent Systems
}

\author{
Chern Han Yong* and Risto Miikkulainen \\ Department of Computer Sciences \\ The University of Texas at Austin \\ Austin, TX 78712 USA \\ yongch, ristodcs.utexas.edu
}

Technical Report AI07-338

\begin{abstract}
In certain tasks such as pursuit and evasion, multiple agents need to coordinate their behavior to achieve a common goal. An interesting question is, how can such behavior be best evolved? A powerful approach is to control the agents with neural networks, coevolve them in separate subpopulations, and test them together in the common task. In this paper, such a method, called Multi-Agent ESP (Enforced SubPopulations), is proposed and demonstrated in a prey-capture task. First, the approach is shown more efficient than evolving a single central controller for all agents. Second, cooperation is found to be most efficient through stigmergy, i.e. through role-based responses to the environment, rather than direct communication between the agents. Together these results suggest that role-based cooperation is an effective strategy in certain multi-agent domains. [ This paper is a revision of AI01-287. ]
\end{abstract}

\section{Introduction}

In cooperative multi-agent problem solving, several agents work together to achieve a common goal. Due to their distributed nature, multi-agent systems can be more efficient, more robust, and more flexible than centralized problem solvers. A central issue with such systems is how such cooperation can be best established. First, should the agents be implemented as a diverse set of autonomous actors, or should they be coordinated by a central controller? Second, if the agents are autonomous, what kind of communication is necessary for them to cooperate effectively in the task?

In this paper, these issues are addressed from the machine learning perspective: A team of neural networks is evolved using genetic algorithms to solve a cooperative problem. The Enforced SubPopulations method of neuroevolution (ESP $[10,11]$ ), which has proven highly efficient in single-agent reinforcement learning tasks, is first extended to multi-agent evolution, in a method named Multi-agent ESP. This method is then evaluated in a pursuit-evasion task where a team of several predators must cooperate to capture a fast-moving prey. The main contribution is to show how different ways of encoding, evolving, and coordinating a team of agents affect performance in the task. Two hypotheses are tested: First, a coevolutionary approach (using Multi-agent ESP), where autonomous neural networks are evolved cooperatively to each control a single predator of the team, is shown to outperform a central-controller approach, where a single neural-network is evolved (using ESP) to control the entire team. This is because niching in coevolution,

*Present address: Institute for Infocomm Research, Singapore 119613, chyong@i2r.a-star.edu.sg 
which is especially strong in ESP [10,11], can be further leveraged in Multi-agent ESP to meet the demands of multi-agent tasks. Instead of searching the entire space of solutions, coevolution allows identifying a set of simpler subtasks, and optimizing each team member separately and in parallel for one such subtask.

Second, the agents do not even need to communicate directly with each other to behave cohesively: It is sufficient that they coevolve to establish compatible roles mediated through stigmergy, that is, they learn to react to environmental changes caused by the teammates. In fact, when a primitive form of direct communication is available (where each team member broadcasts its location to its teammates), communicating teams consistently perform worse than teams without direct communication! Each agent learns to perform its subtask (or role) reliably, and the task is solved through the stigmergic interaction of these roles; direct communication is unnecessary and only complicates the task.

The paper will begin with a brief review of related work in cooperative coevolution, multi-agent learning, and the prey-capture domain. The Multi-Agent Enforced SubPopulations method is then described, followed by its experimental evaluation. A discussion of future prospects of this approach concludes the paper.

\section{Background and Related Work}

The term cooperation is used in two distinct ways in this paper. The first refers to a phenomenon of multiagent behavior where agents work together to achieve a common goal, as will be discussed in Section 3 . The second refers to cooperation as a mechanism for learning, and will be discussed in detail in Section 2.1. Section 2.2 will discuss agent communication through stigmergy.

\subsection{Cooperative Coevolution}

Coevolution in Evolutionary Computation means maintaining and evolving multiple individuals in a common task, either in a single population or in multiple populations. In competitive coevolution, these individuals have adversarial roles in that one agent's loss is another one's gain. In cooperative coevolution, however, the agents share the rewards and penalties of successes and failures. It is most effective when the solution can be naturally modularized into components that interact, or cooperate, to solve the problem. Each component can then be evolved in its own population, and each population contributes its best individual to the solution.

For example, Gomez and Miikkulainen [10] developed a method called Enforced SubPopulations (ESP) where populations of neurons are evolved to form a neural network. A neuron is selected from each population to form the hidden layer of the network, which is then evaluated on the problem and its fitness passed back to the participating neurons. In the multi-agent evolution developed in this paper, ESP is used to evolve each neural network, but the networks are also required to cooperate. The ESP method and the multi-agent extension to it are discussed in more detail in Section 4.

Potter and De Jong [31] designed an architecture and process of cooperative coevolution similar to ESP, focusing on methodological issues such as how to decompose the problem automatically into an appropriate number of subcomponents and roles. In contrast, the focus of this paper is on understanding cooperation itself, including the roles of team control and communication.

In a series of papers, Haynes and Sen [16, 14, 15] explored various ways of encoding, controlling, and evolving predators that behave cooperatively in the prey-capture domain. In the first of these studies [14], Genetic Programming was used to evolve a population of strategies, where each individual was a program that represented the strategies of all predators in the team. The predators were thus said to be homoge- 
neous, since they each shared the same behavioral strategy. In follow-up studies $[16,15]$, they developed heterogeneous predators: Each chromosome in the population was composed of $k$ different programs, each one representing the behavioral strategy of one of the $k$ predators in the team. Haynes and Sen reported that the heterogeneous predators were able to perform better than the homogeneous ones. In this paper, heterogeneity is taken a step further by evolving the controllers for each predator in separate populations.

In contrast to the advantage found for heterogeneous teams in the above studies, Luke [21] observed that heterogeneous teams could not outperform homogeneous teams evolved using Genetic Programming in the soccer softbot domain. However, he conjectured that, given sufficient time, the heterogeneous approach would have learned better strategies. Such time was not practical in his domain, where each evaluation cycle took between 20 seconds and one minute.

Quinn et al. [32] and Baldassarre et el. [3] evolved teams of homogeneous robots controlled by neural networks, and studied how the role allocations emerge in collective behaviors such as formation movement and flocking. In contrast, this paper shows how roles are allocated in heterogeneous teams of agents, focusing in particular on the effects of global vs. global control and communication.

Balch [2] examined the behavioral diversity developed by robot teams using reinforcement learning. He found that when the reinforcement was local, i.e. applied separately to each agent, the agents within the team learned identical behaviors; global reinforcement shared by all agents, on the other hand, produced teams with heterogeneous behavior. This result provides a useful guideline for evolving cooperating agents: Rewarding the whole team for good behavior privileges cooperation even when some agents do not contribute as much as others, whereas rewarding individuals induces more competitive behaviors because each individual tries to maximize its own reward at the expense of the good of the entire team. The work reported in this paper diverges from Balch's in focus and implementation: The focus is on how global versus local control and communication affect the diversity and niching of the behaviors that evolve, and instead of reinforcement learning, the implementation is based on evolutionary learning of neural networks [11].

\subsection{Agent Communication}

The role of communication in cooperative behavior has been studied in several Artificial Life experiments [41, 5, 29, 19, 37]. These studies showed that communication can be highly beneficial, even crucial, in solving certain tasks. However, the cost of communication--such as the energy expenditure in signaling, or the danger of attracting predators, or the complexity of the apparatus required-was not taken into account in most of these studies. In fact, even in domains where communication does contribute toward solving a task, communicative traits may still not evolve if they involve a significant cost [39]. Other kinds of cooperative strategies may evolve instead, depending on the nature of the task, how dense the population is, and whether resources are available.

One particularly interesting form of such cooperation without communication is stigmergy, a concept proposed by Grassé [12] to describe the indirect communication observed in the behaviors of certain social insects. Grassé observed that worker termites were stimulated to perform certain activities by a particular configuration of their nest, transforming it into a new configuration, which would in turn stimulate other activities. The word stigmergy was coined to describe this process: "The stimulation of the workers by the very performances they have achieved is a significant one inducing accurate and adaptable response, and has been named stigmergy" [12] (translated by Holland and Melhuish [18]).

Holland and Melhuish [18] examined stigmergy and self-organization in a group of robots that clustered and sorted frisbees, and found that the task was solvable using stigmergy-based control without any direct communication between robots. Franklin [8] proposed that stigmergic coordination may be an advantage over coordination through direct communication, because communication and explicit planning between 
agents require additional architecture, intelligence, and resources. The work presented in this paper further advances these ideas in three ways: (1) By showing how stigmergy can emerge in cooperative coevolution when direct communication between teammates is not available; (2) by comparing the learning performance of communicating teams and those that coordinate through stigmergy; and (3) by comparing the emergent behaviors of communicating teams and those that coordinate through stigmergy. As a result, an effective non-communicating strategy based on stigmergic coordination is identified and termed role-based cooperation. In certain tasks, this strategy is easier to learn and more powerful than a strategy based on communication.

In this paper, a most elementary form of communication is employed. Whereas each non-communicating agent is completely unaware of its teammates, each communicating agent continuously broadcasts its location to its teammates. Although elementary, such exchange of information is useful communication: In many real-world applications of software agents or physically situated robots, it is not possible to sense the teammates locations directly, because they may be far away or obscured or the appropriate sensors may not be available. A communicative apparatus (such as a radio system) is required to obtain this information. On the other hand, while other, more complex forms of communication are possible, broadcasting of locations is sufficient to demonstrate differences between teams that communicate and those that do not, and it therefore forms a suitable experimental platform for this study, as will be described next.

\section{The Prey-Capture Domain}

The prey-capture task used in this paper is a special case of pursuit-evasion problems [24]. Such tasks consist of an environment with one or more preys and one or more predators. The predators move around the environment trying to catch the preys, and the preys try to evade the predators. Pursuit-evasion tasks are interesting because they are ubiquitous in the natural world, offer a clear objective that allows measuring success accurately, and allow analyzing and visualizing the strategies that evolve. Such tasks generally cannot be solved with standard supervised learning techniques like backpropagation. The correct or optimal decisions at each point in time are usually not known, and the performance can be measured only after several decisions have been made. More complex algorithms are required that can learn sequences of decisions based on sparse reinforcement. Pursuit-evasion tasks are challenging for even the best learning systems because they require coordination with respect to the environment, other agents with compatible goals, and adversarial agents [10].

The prey-capture task in this paper consists of one prey and three predators in a discrete toroidal environment (Figure 1). The prey is controlled by a rule-based algorithm; the predators are controlled by neural networks. The goal is to evolve the neural networks to form a team for catching the prey. The different approaches and techniques are compared based on how long it takes for the team to learn to catch the prey consistently, and what kind of strategies they use.

The environment is a $100 \times 100$ toroid without obstacles or barriers (the $100 \times 100$ area is also referred to as the "world" below). All agents can move in 4 directions: N, S, E, or W. The prey moves as fast as the predators, and always directly away from the nearest predator. It starts at a random location of the world, and the predators start in a horizontal row at the bottom left corner (Figure 1a). All the agents make their moves simultaneously, and an agent can move into a position occupied by another agent. The team catches the prey when a predator moves onto the position occupied by the prey. If the predators have not caught the prey in 150 moves, the trial is terminated and counted as a failure.

Constrained in this way, it is impossible to consistently catch the prey without cooperation. First, since the predators always start at the bottom left corner, behaving greedily would mean that they chase the prey 


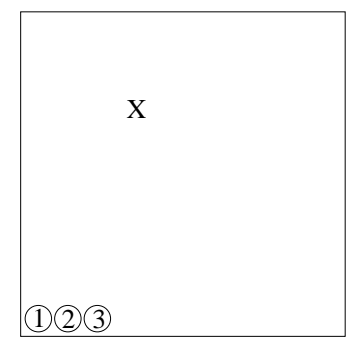

(a)

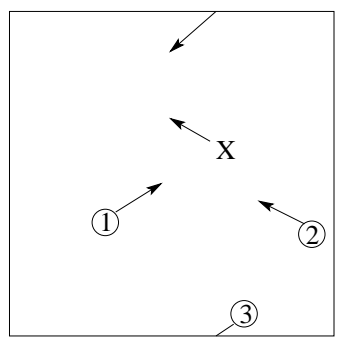

(b)

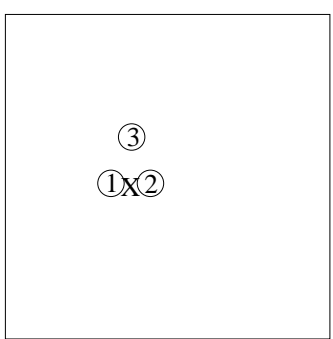

(c)

Figure 1: The Prey-Capture Domain. The environment is a $100 \times 100$ toroidal grid, with one prey (denoted by "X") and three predators (denoted by "1", "2" and "3"). Figure (a) illustrates a starting scenario: The predators start in a row at the bottom left corner, and the prey starts in a random location. Figure (b) illustrates a scene later during a trial. The arrows indicate a general direction of movement: Since each agent may only move in the four cardinal directions, a movement arrow pointing 45 degrees northwest means the agent is moving north and west on alternate time steps. Figure (c) shows the positions of the predators one time step before a successful capture. Even though the prey is as fast as the predators, the predators approach it consistently from different directions, and eventually the prey has nowhere to run.

as a pack in the same direction. The prey will then avoid capture by running away in the same direction: Because it is as fast as the predators, and the environment is toroidal, the predators will never catch it (Figure 18 demonstrates this scenario). On the other hand, should the predators behave randomly, there is little chance for them to approach, circle, and run into the prey. The 150 steps limit is chosen so that the predators can travel from one corner of the world to the other, i.e. they have enough time to move to surround the prey, but it is not possible for them to just mill around and capture the prey eventually by accident.

The prey-capture task has been widely used to test multi-agent behavior. For example, Benda et al. and Haynes and Sen $[6,16,15]$ used this domain to assess the performance of different coordination systems, and Jim and Giles [19] studied the evolution of language and its effect on performance. In their variant of the task, the predators are required to surround the prey in specific positions to catch it, and the main difficulty is in coordinating the predators to occupy the proper capture positions simultaneously. On the other hand, the prey moves either randomly or at a slower speed than the predators, thus allowing the predators to catch up with it easily. In contrast, in the experiments described in this paper it is enough for one predator to move onto the prey to capture it. However, the prey moves as fast as the predators, and always away from the nearest predator, and therefore there is no way to catch the prey simply by chasing it. The main challenge is in coordinating the chase: The agents have to approach the prey from different directions so that it has nowhere to go in the end (Figure 1c). This behavior requires developing a long-term cooperative strategy, instead of coordinating the timing of a few actions accurately as in the previous studies.

\section{The Multi-Agent ESP Approach}

The Enforced SubPopulations Method $\left(\mathrm{ESP}^{1} ;[10,11]\right)$ is an extension of Symbiotic, Adaptive NeuroEvolution (SANE; [26, 27, 25]). SANE is a method of neuro-evolution that evolves a population of neurons instead of complete neural networks. In other words, in SANE each chromosome represents the connections of a single neuron instead of the structure and weights of an entire network (analogous to the "Michigan" method of evolving rule-based systems, where each chromosome represents a single rule [17], versus the entire rule set as in the "Pitt" method [36]). Neurons are selected from the population to form the hidden layer of a neural network, which is then evaluated on the problem. The fitness of the network is passed

\footnotetext{
${ }^{1}$ ESP neuroevolution software is available at http://nn.cs.utexas.edu/soft-list.php
} 


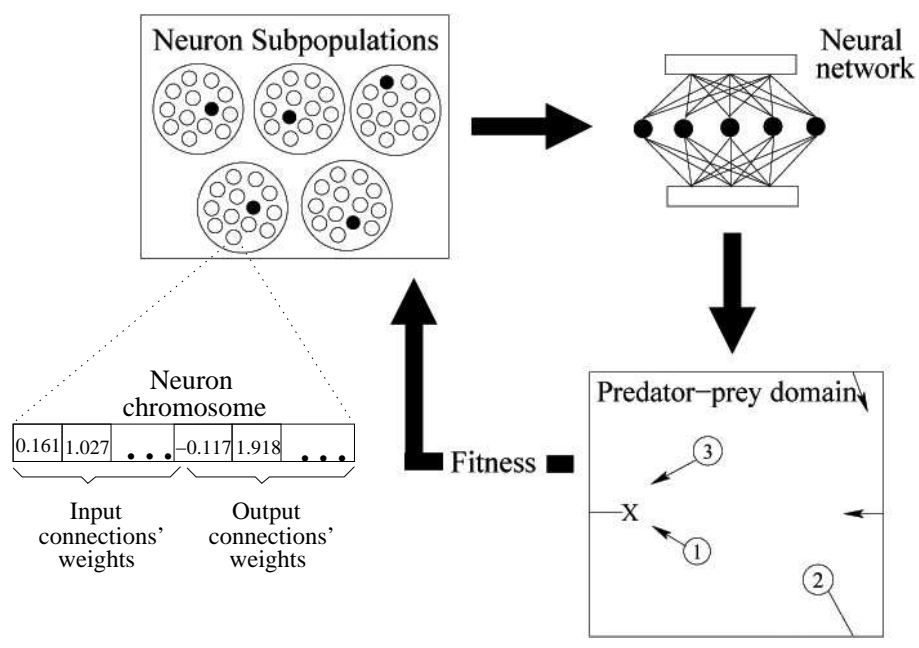

Figure 2: ESP architecture. Each subpopulation of neurons contributes one neuron (with its input and output connections) to form the hidden layer of the neural network, which is then evaluated in the domain. The fitness is passed back to the participating neurons. This scheme is used to evolve the central-controller neural network (Figure 4) that controls all three predators simultaneously. The extension to multiple controllers is shown in Figure 3.

back to all neurons of the network equally. ESP extends SANE by allocating a separate population for each hidden-layer neuron of the network; a number of neuron populations are thus evolved simultaneously (Figure 2). ESP is thus a cooperative coevolution method: Each neuron population tends to converge to a role that results in the highest fitness when the neural network is evaluated. In this way, ESP decomposes the problem of finding a successful network into several smaller subproblems, resulting in more efficient evolution $[10,11]$.

In several robot control benchmark tasks, ESP was compared to other neuro-evolution methods such as SANE, GENITOR [42], Cellular Encoding [13, 43], as well as to other reinforcement learning methods such as Adaptive Heuristic Critic [4, 1], Q-learning [40, 30], and VAPS [23]. Because of its robust neural network representation and efficient search decomposition, ESP turned out to be consistently the most powerful, solving problems faster, and solving harder problems [11,9]. The above properties of ESP also allow it to be extended efficiently to multi-agent systems evolution. In this paper, ESP is extended to simultaneous evolution of multiple agents, called Multi-agent ESP.

Three approaches of evolving and controlling agents are tested: the central controller approach, the autonomous communicating approach, and the autonomous non-communicating approach. In the central controller approach, all three predators are controlled by a single feedforward neural network, implemented with the usual ESP method (Figure 2). Multi-agent ESP is used in both the communicating and non-communicating autonomous controllers approaches, where each predator is controlled by its own feedforward network, evolved simultaneously in separate populations (Figure 3). Each network is formed using the usual ESP method. These three networks are then evaluated together in the domain as a team, and the resulting fitness for the team is distributed among the neurons that constitute the three networks.

In all three approaches, the agents are evolved in a series of incrementally more challenging tasks. Such incremental evolution, or shaping, has been found to facilitate learning in complex domains, where direct evolution in the goal task would result in inadequate, mechanical strategies such as running around in circles $[10,34,7,38]$. Evolution proceeds through six stages: In the easiest task the prey is stationary, and in each subsequent task it moves at a faster speed and with a greater probability of heading away from the nearest predator, until in the final task it moves as fast as the predators, and always away from the nearest predator. 


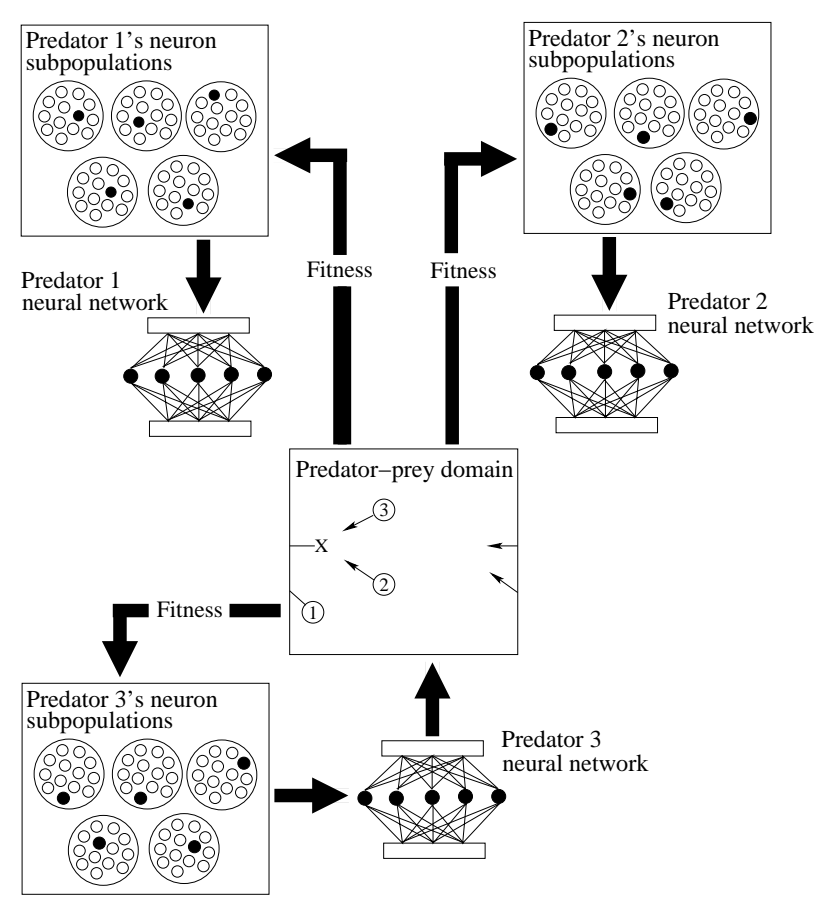

Figure 3: Multi-agent ESP architecture. Each predator is controlled by its own neural network, formed from its own subpopulations of neurons. The three neural networks are evaluated in the domain at the same time as a team, and the fitness for the team is passed back to all participating neurons. A detailed architecture for the individual networks is shown in Figure 5.

\begin{tabular}{|c|c|c|}
\hline Task & Prey Speed (Relative to Predators' Speeds) & Prey Probability of Moving Away from Nearest Predator \\
\hline $\mathbf{1}$ & 0 & 0 \\
\hline $\mathbf{2}$ & 0.45 & 0.45 \\
\hline $\mathbf{3}$ & 0.63 & 0.63 \\
\hline $\mathbf{4}$ & 0.77 & 0.77 \\
\hline $\mathbf{5}$ & 0.89 & 0.89 \\
\hline $\mathbf{6}$ & 1 & 1 \\
\hline
\end{tabular}

Table 1: The sequence of incrementally more difficult tasks.

Table 1 gives the speeds and evasive probabilities of the prey for each of these tasks; small variations to this schedule lead to similar results. When a team manages to solve the current task consistently, the next harder task is introduced; the team can thus utilize what it has already learned in the easier task to help guide its learning in the new, harder task. In the pursuit-evasion domain, incremental evolution is particularly useful because it allows the predators to learn early on how to catch the prey at close proximity. Placing the predators into the final task right from the start fails because they do not get close to the prey often enough to learn to catch it. Incremental learning is therefore used to give evolution more experience in the necessary skills that would otherwise be hard to develop.

The fitness function consists of two components, depending on whether the prey was captured or not:

$$
f= \begin{cases}\frac{d_{0}-d_{e}}{10} & \text { if the prey was not caught } \\ \frac{200-d_{e}}{10} & \text { if the prey was caught, }\end{cases}
$$

where $d_{0}$ is the average initial distance of the predators from the prey, and $d_{e}$ is the average final distance. 
This fitness function was chosen to satisfy four criteria:

1. If the prey is caught, the starting scenario (i.e. the initial distance from the prey) should not bias the fitness. Instead, teams should be rewarded if their ending positions are good-that is, if all predators are near the prey.

2. If the prey is not caught, teams that covered more distance should receive a higher reward.

3. Since a successful strategy has to involve sandwiching the prey between two or more predators, at least one predator must travel the long distance of the world so that two predators can be on the opposite sides of the prey. Thus the time taken for each capture (within the 150 step limit) tends to be about the same, and should not be a factor in the fitness function.

4. The fitness function should have the same form throughout the different stages of incremental evolution, making it simple and convenient to track progress.

The neuron chromosomes are concatenations of the real-valued weights on the input and output connections of the neuron (Figure 2). As is usual in ESP, burst mutation through delta-coding [44] on these weights is used as needed to avoid premature convergence: If progress in evolution stagnates (i.e. the best solution 25 generations earlier outperforms the current best solution), the populations are re-initialized according to a Cauchy distribution around the current best solution. Burst mutation typically takes place in prolonged evolution in difficult tasks $[10,11]$.

\section{Evolution of Cooperative Behavior}

In this section, two experiments are presented, testing the two main hypotheses of this paper: First, that cooperative coevolution of autonomous controllers is more effective than evolving a central controller in this domain (Section 5.2), and second, that the agents controlled by autonomous neural networks can learn to cooperate effectively using stigmergy without any direct communication (Section 5.3). These behaviors and conditions under which they arise are then analyzed in detail in Sections 6 and 7.

\subsection{Experimental Setup}

The following parameter settings were used for ESP and its multi-agent extension. Each subpopulation of neurons consisted of 100 neurons; each neuron (or chromosome) was a concatenation of real-valued numbers representing full input and output connections of one hidden unit. During each evolutionary generation, 1,000 trials were run wherein the neurons were randomly chosen (with replacement) from their subpopulations to form the neural network(s). In each trial, the team was evaluated nine times to match the number of evaluations in the test benchmark suite (to be described shortly). The prey started in a random location each time, while the predators always started in the bottom-left corner (Figure 1a). The consistent starting location gives the different trials a common structure that makes it easier to analyze and compare results; Section 7.3 shows that similar results are obtained when predators start at random locations. The fitnesses over the nine evaluations are averaged, and assigned to all the neurons that constituted the network. After the trials, the top $25 \%$ of neurons were recombined using one-point crossover. The offspring replaced the bottom $50 \%$ of the neurons, and they were then mutated with a probability of 0.4 on one randomly-chosen weight on each chromosome, by adding a Cauchy-distributed random value to it. Small changes to these parameters lead to similar results. 


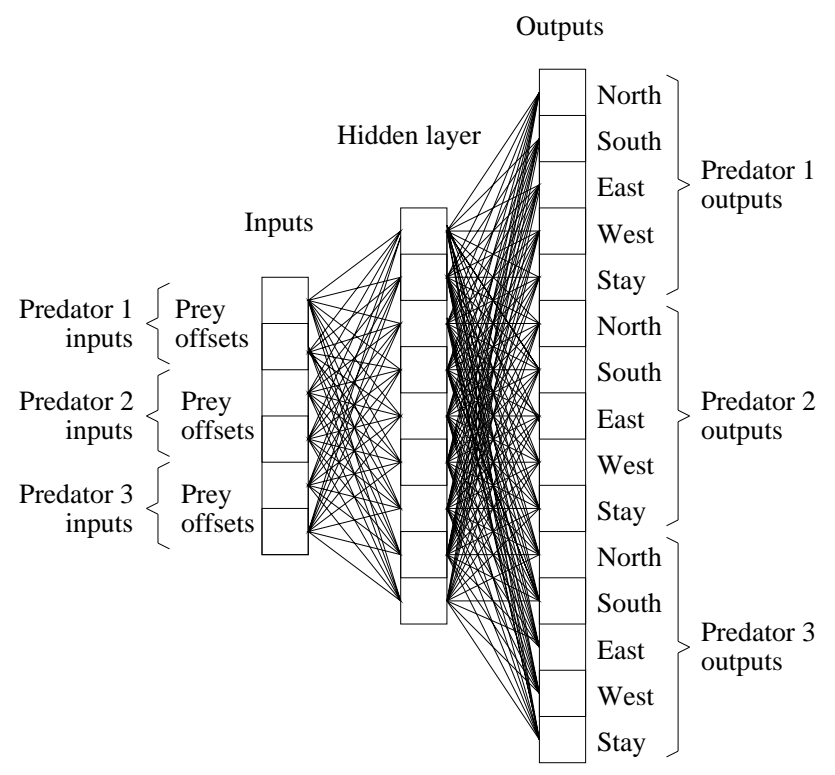

Figure 4: Central controller network for a team of three predators. This network receives the relative $x$ and $y$ offsets of the prey from the perspective (i.e. location) of all three predators, and outputs the movement decisions for all three predators. This way it acts as the central controller for the whole team. There are nine hidden units, and the chromosomes for each hidden layer unit consist of 21 real-valued numbers (six inputs and 15 outputs).

The environment is stochastic only in the prey's starting location, and this location is the only factor that determines the course of action taken by the predators. In order to test these team strategies comprehensively, a suite of benchmark problems was implemented. The world was divided into nine $33 \times 33$ subsquares; in each trial, each team was tested nine times, with the prey starting at the center of each of these squares in turn. Such an arrangement provides a sampling of the different situations, and allows estimating how effective each team is in general. A team that manages to catch the prey in all nine benchmark cases is considered to have completely solved the task, and indeed such a team usually has a $100 \%$ success rate in random, general scenarios.

Before running the comparisons, an appropriate number of hidden units was determined for each of the three approaches. Since small networks typically generalize better and are faster to train [20, 33], the smallest number of hidden units was found that allowed solving the task reliably. More specifically, for each of the three approaches, ten evolution runs were performed on the prey-capture task, initially with two hidden units. Should any of the ten runs fail to learn to solve the task completely, the number of hidden units was increased by one and another ten runs were tried. A run was deemed a failure if it stagnated ten consecutive times, that is, if its fitness did not improve despite ten burst mutations in 250 generations.

Through this procedure, the appropriate number of hidden units was determined to be nine for the central controller, eight for each of the autonomous communicating controllers, and three for each of the autonomous non-communicating controllers (Figures 4-6). The comparisons were run with these architectures, as described below.

\subsection{Standard Evolution of a Central Controller vs. Cooperative Coevolution of Autonomous Controllers}

This section tests the first hypothesis, i.e. that it is easier to coevolve three autonomous communicating neural networks, each controlling a single predator (Figure 5), than it is to evolve a single neural network 


\section{Hidden layer}

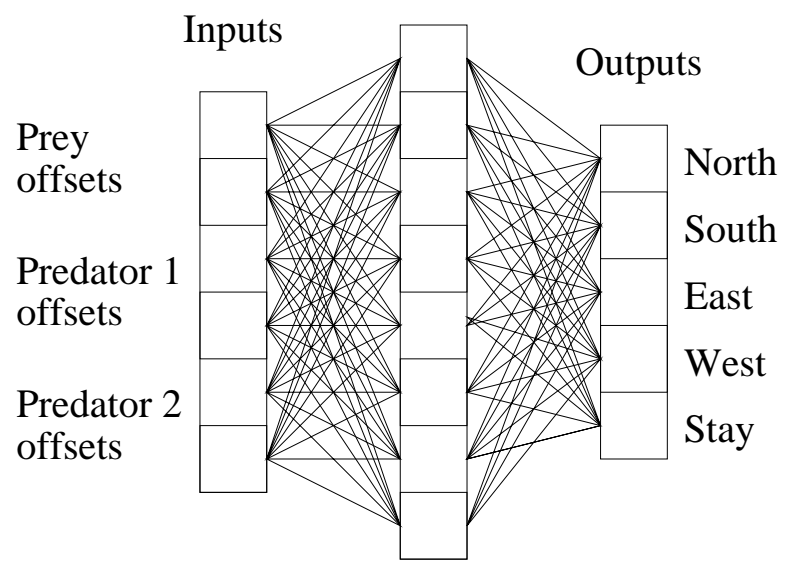

Figure 5: Controller for each autonomous communicating predator. This network autonomously controls one of the predators; three such networks are simultaneously evolved in the task. The locations of this predator's teammates are obtained, and their relative $x$ and $y$ offsets are calculated and given to this network as information obtained through communication. It also receives the $x$ and $y$ offsets of the prey. There are eight hidden units, and the chromosomes for each hidden layer unit consist of 11 real-valued numbers (six inputs and five outputs).

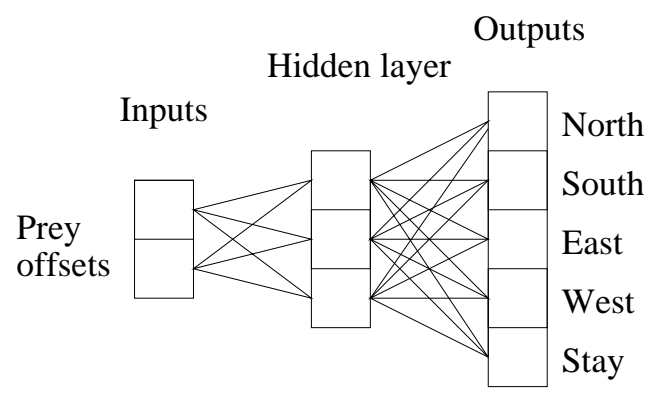

Figure 6: Controller for each autonomous non-communicating predator. This network receives the prey's $x$ and $y$ offsets as its inputs. Therefore, it controls a single predator without knowing where the other two predators are (i.e. there is no communication between them). There are three hidden units, and the chromosomes for each hidden layer unit consist of seven real-valued numbers (two inputs and five outputs).

that controls the entire team (Figure 4). The number of evolutionary generations needed to learn to solve the task, that is, to be able to catch the prey in all nine benchmark cases, are compared for the two approaches. Ten simulations with different random number seeds were run in each case.

Figure 7 shows a clear result: On average, the three autonomous controllers were evolved almost twice as fast as the centralized controller. The conclusion is that the cooperative coevolution approach is more powerful than the centralized approach in this task.

Figure 8 shows how long it took each approach to solve each incrementally more difficult task during evolution. While the centrally controlled teams require just slightly more time to learn the easier tasks than the autonomous controllers, as the tasks become more difficult, the differences in performance grow significantly. This result suggests that the autonomous controller approach should scale up better to harder tasks. 


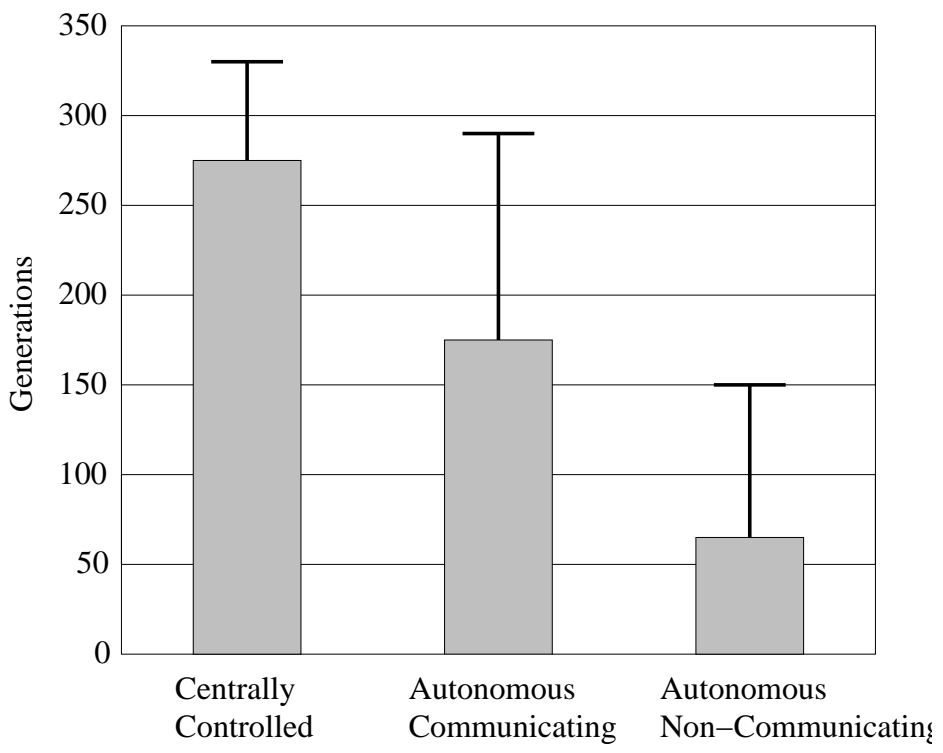

Figure 7: Learning performance for each approach. The average number of generations, with standard deviation, required to solve the task is shown for each approach. The centrally controlled team took 50\% longer than the autonomously controlled, communicating team, which in turn took over twice as long as the autonomously controlled, non-communicating team to learn the task. All differences are statistically significant with $p>0.95$.

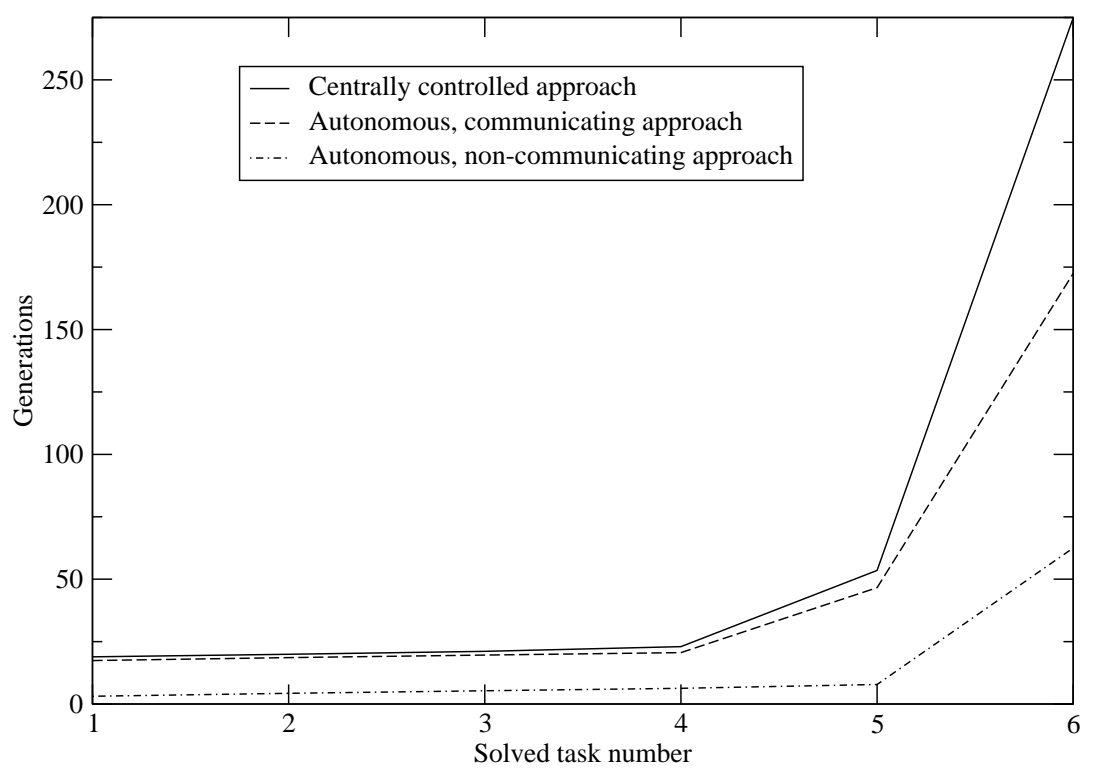

Figure 8: Progress of evolution through incrementally more difficult tasks. Number of generations required for each approach to solve each task in the sequence is shown. As shown in Table 1, in Task 1 the prey is stationary, whereas in Task 6 it moves at the same speed as the predators, and always away from the nearest predator. The centrally controlled teams took only slightly longer than the autonomously controlled communicating teams to learn the easier tasks; with more difficult tasks, though, the differences in performance become significant. On the other hand, the autonomously controlled, non-communicating teams learn to solve all the tasks substantially faster than either of the two other approaches. 


\subsection{Cooperative Coevolution With vs. Without Communication}

The conclusion from the first comparison is that separating the control of each agent into disjoint autonomous networks allows for faster evolution. The controllers no longer receive direct information about what the other agents see; however the domain is still completely represented in each predator's inputs, which include the relative locations of the teammates and the prey. In this section the available information is reduced further by preventing the predators from knowing each other's locations. This way the agents will have to act independently, relying on stigmergy for coordination. The objective is to test the second hypothesis, i.e. that cooperative behavior based on stigmergy may evolve more efficiently than cooperation based on direct communication.

The network architecture for such non-communicating controllers is shown in Figure 6. The predator no longer receives the relative $x$ and $y$ offsets of the other predators, only the offsets of the prey. These networks were evolved with the same coevolutionary multi-agent ESP method as the communicating networks of Figure 5. Again, ten simulations were run and the results averaged.

The non-communicating teams learned to solve the entire task more than twice as fast as the communicating teams (Figure 7). Furthermore, the non-communicating teams learned to solve each incrementally more difficult task significantly faster than the communicating teams (Figure 8).

These results show that direct communication between teammates is not always necessary: Cooperative behavior can emerge even when teammates are unaware of each other's locations. In fact, since communication is not necessary, it is more efficient to do away with it entirely. In the next section examples of evolved behaviors will be analyzed to gain insight into why this is the case, concluding that the agents rely on stigmergy. In Section 7, a series of further simulations will be presented to demonstrate that this result is robust; in particular, Section 7.1 shows that this result is not due to the relative differences in search space between the two approaches.

\section{Analysis of Evolved Behaviors}

In this section, the behaviors evolved in the three approaches are analyzed in two ways: First, the degree to which each predator's actions depend on those of the other predators is measured quantitatively; second, examples of evolved behaviors are analyzed qualitatively. This analysis leads to the characterization of team behaviors in two extremes: those based on role-based cooperation, where interaction between different roles take place through stigmergy, and those based on direct communication.

\subsection{Measuring Dependency}

A predator's actions are independent of its teammates if the predator always performs the same action for the same prey position, regardless of where its teammates are. Conversely, the predator's strategy is dependent on its teammates if its actions are determined by both the prey's and the teammates' positions.

The team of autonomous, non-communicating predators must act independently: Since the neural network for each predator cannot see the locations of its teammates, their positions cannot affect its actions. A more interesting question is whether dependent actions evolve in the communicating team. The agents could evolve to simply ignore their communication inputs; on the other hand, they could learn to use communication to develop a coordinated strategy. Also, it is interesting to observe whether the actions of a centrally controlled team are more dependent than those of an autonomous communicating team, since its control architecture is more tightly coupled between teammates. 


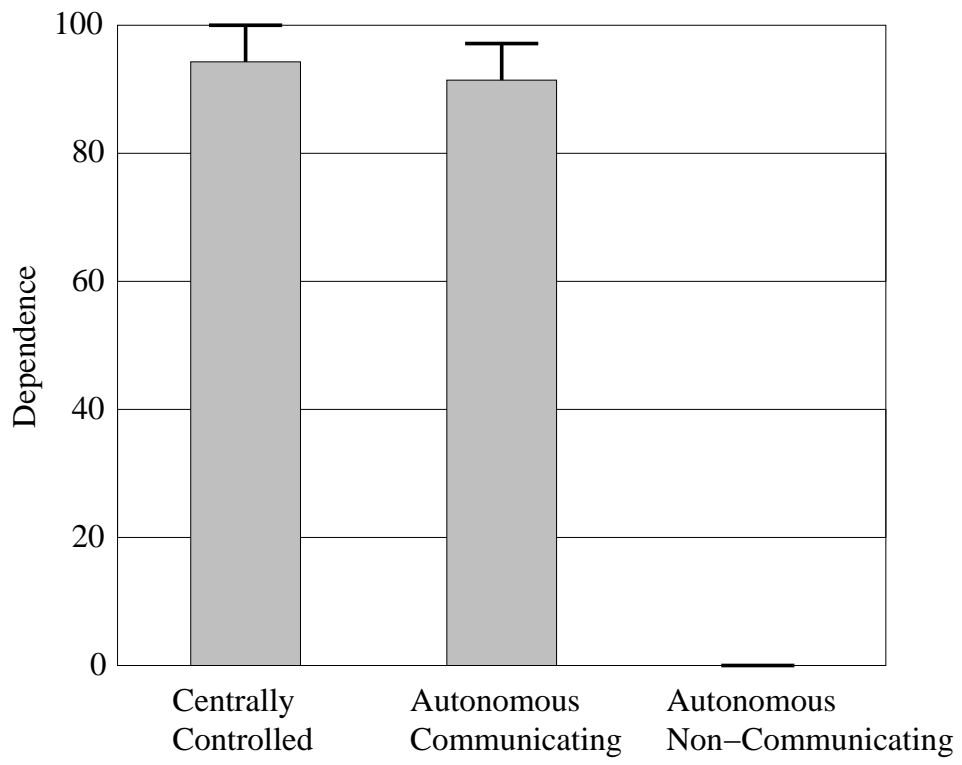

Figure 9: Action dependence in teams evolved using each approach. The centrally controlled and autonomous communicating teams both evolved behaviors in which each agent's actions were highly dependent on its teammates (the difference is not statistically significant, with $p=0.84$ ). The non-communicating team evolved behaviors that were independent (the difference between the dependence of the non-communicating team and those of the other two approaches is statistically significant at $p>>0.99$ ).

Action dependence can be measured in the following way: For each predator in the team, a sample is taken of possible relative prey positions. For each of these sampled positions, possible configurations of teammates' locations are then sampled. Each such case is presented to the predator's control network, and the predator's resulting action observed. The percentage of actions that differ for the same prey position (but different teammate positions) is a measure of how dependent this predator's actions are on its teammates. The team's dependence is obtained as the average of those of its three predators.

The dependences of the centrally controlled, communicating, and non-communicating teams are compared in Figure 9. As expected, the non-communicating predators act independently. In contrast, over 90\% of the actions of the centrally controlled and communicating team members depend on the other predators. On average, the centrally controlled teams were slightly more dependent than the communicating teams; however, this difference is not statistically significant.

These results demonstrate that indeed the communicating teams learn a distinctly different behavior than the non-communicating teams. Even though the weights on the communication inputs could evolve to 0 , they do not. Apparently, given a starting point where all weights are random, it is easier for evolution to discover an adequate communicating strategy than unlearn communication altogether. An interesting issue is whether a different starting point would bias evolution to discovering non-communicating solutions instead; this question is addressed in Section 7.4.

\subsection{Characterizing Sample Behaviors}

Dependence measurements demonstrate that the behaviors differ, but to understand how, actual examples need to be analyzed. In this section, example behaviors for each approach are described and compared in detail. The main results are that without communication, evolution produces specific roles for each team member, and these roles interact only indirectly through stigmergy, that is, by causing changes to 


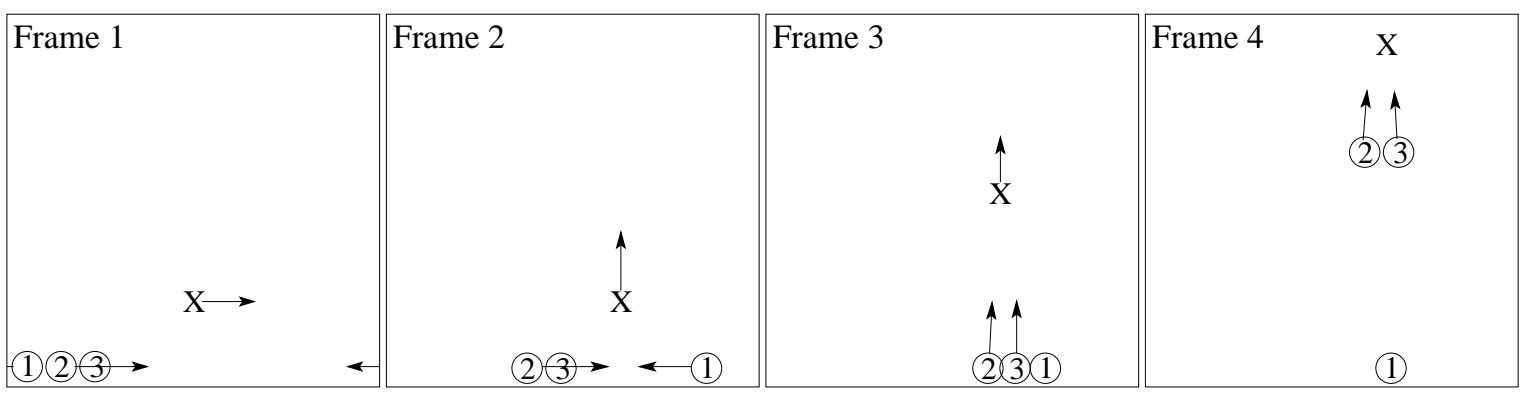

Figure 10: A sample strategy of a non-communicating team. In frames 1 and 2, the predators are in setup mode, maneuvering into an appropriate chase configuration. In frame 3, they switch to chase mode: Predators 2 and 3 chase the prey toward predator 1, which acts as a blocker. This strategy is effective and does not require direct communication. Animated demos of this strategy, and others discussed in this paper, are available at nn.cs.utexas.edu/keyword?mesp-demo

the environment that affect the other teammates' roles; furthermore, these teams utilize a single effective strategy in all cases. On the other hand, evolution with communication produces agents with more variable (although less effective) behaviors, able to employ two or more different strategies at different times.

A typical successful strategy for the non-communicating team is illustrated in Figure 10. This strategy is composed of two stages, the setup stage and the chase stage. In the setup stage, illustrated in the first two frames, the predators maneuver the prey into an appropriate configuration for a chase: In frame one, predators 2 and 3 move eastward, causing the prey to flee in the same direction, while predator 1 moves westward. When the prey detects that predators have closed in to its south, it starts fleeing northward (frame two). In frame three, the predators detect that the prey is directly to their north, and the chase stage of the strategy begins. This stage involves two different roles, chasers and blockers. Predator 1, the blocker, moves only in the horizontal direction, staying on the same vertical axis as the prey, while predators 2 and 3, the chasers, pursue the prey northward (frames three and four). Eventually, the prey is trapped between the blocker and the chasers, who move in for the capture. Notice that this strategy requires no direct communication between predators: As long as the predators get the prey into a chase configuration, and the blockers and chasers execute their roles, the prey will always be caught. Moreover, it is reliable for all starting locations of the prey, even when the predators do not all switch from setup to chase modes at the same time.

From the above description, it is clear that some form of coordination is taking place, even without direct communication. When the prey is not directly above (or below) a predator, the predator is in setup mode, moving either east (predators 2 and 3), or west (predator 1). This action causes a change in the environment, i.e. a specific reaction on the part of the prey: to flee east or west. Because of this reaction, each of the predators will eventually find the prey directly above or below it, triggering a second activity: They either chase the prey north (predators 2 and 3), or remain on the prey's vertical axis (predator 1). This activity in turn causes a change in the prey's behavior, to flee north, until it is eventually trapped between chasers and blockers. This sequence of events is a clear example of stigmergy: Each agent's action causes a change in the environment, that is, a reaction on the part of the prey; this reaction in turn causes a change in the agent's and its teammates' behaviors.

Sample behavior of a communicating team is illustrated in Figure 11. Two different strategies are shown because this team actually displays both of them, and also their combinations and variations, depending on the relative locations of the prey and the other predators at each timestep. The first strategy, Figure 11a, illustrates behavior similar to the chaser-blocker strategy. The first frame is a snapshot of the starting posi- 
(a)

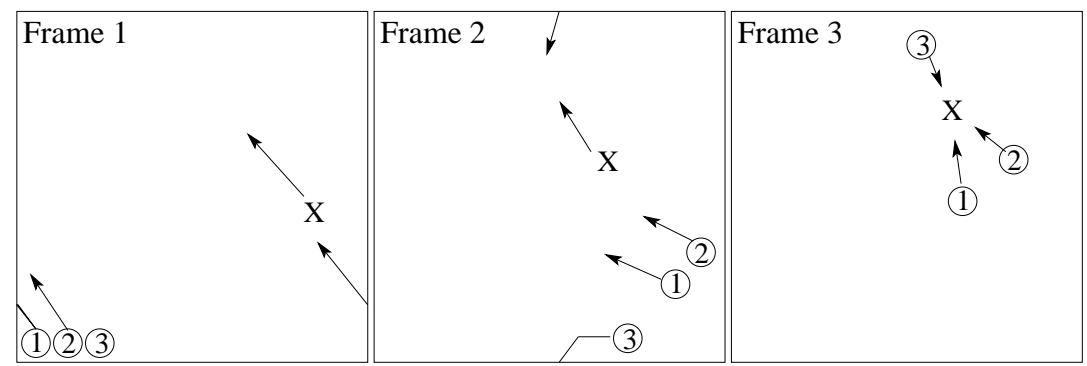

(b)

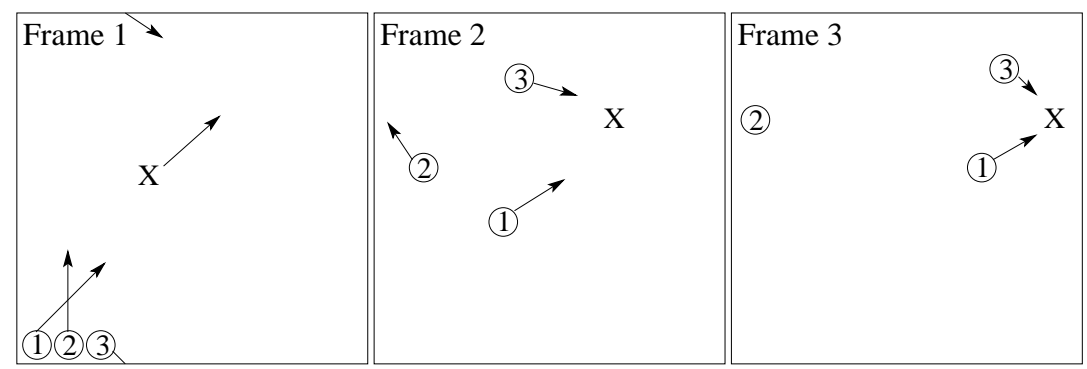

Figure 11: Two sample strategies of the same communicating team. This team employs the two strategies shown above, as well as their variations and combinations. In the first, (a), the chase starts with two chasers and a blocker, but ends with opposite chasers. In the second, (b), there is a blocker and two chasers throughout, but the movement is horizontal. In this manner, the same team utilizes different strategies, depending on the starting position of the prey.

tion. Predators 1 and 2 are the chasers, and they start pursuing the prey upward. Predator 3 is the blocker, and it moves left onto the prey's vertical axis. At this point, however, it starts chasing the prey downward, in Frame 2, until the prey is trapped between all three predators in Frame 3. Already this strategy is more varied as those of the non-communicating teams, as a combination of blocking and opposite chasers.

Another strategy employed by the same team in a different situation is shown in Figure $11 b$. In the first frame, predators 1 and 3 start moving toward the prey diagonally upward and downward, while predator 2 moves upward until it is horizontal with the prey. By the second frame, predators 1 and 3 are chasing the prey horizontally, until it is trapped between them and predator 2 in frame 3 . This strategy is again similar to the chaser-blocker strategy, except this time the prey is chased horizontally instead of vertically, and the chase includes diagonal movement as well.

Although each strategy is similar to those of non-communicating teams, in this case they are employed by one and the same team. This team occasionally also utilizes combinations of these strategies, for example by starting with one and finishing with another. Thus, each predator does not have a specific, fixed role, but modifies its behavior depending on the situation. Through communication each predator is aware of its teammates' relative locations, and its behavior depends not only on the prey's relative position, but also directly on what the other predators are doing. In this way, the communicating strategies are more variable and flexible. On the other hand, they are less efficient to evolve (Section 5.2), and less robust (Section 7.2). In a sense, the non-communicating teams resemble players in a well-trained soccer team, where each player knows what to expect from the others in each play, whereas the behavior of the communicating teams is similar to a pickup soccer team where each player has to constantly monitor the others to determine what to do. Such players can perhaps play with many other kinds of players, but not as efficiently.

The centrally controlled teams exhibit behavior nearly identical to those of the communicating autonomous teams. In particular, the more tightly coupled architecture does not translate to behavior that is visibly more coordinated in this domain. 
Of course we have to be careful not to attribute undue intelligence and intention to neural networks that simply manage to adapt to each other's behavior. However, the differences in behavior are striking: The non-communicating teams employ a single, efficient, fail-proof strategy in which each team member has a specific role, while the communicating and centrally controlled teams employ variations and combinations of two or more strategies. These two forms of cooperative behavior can be distinguished as role-based cooperation and communication-based cooperation. Role-based cooperation consists of two ingredients: Cooperation is achieved through the combination of the various roles performed by its team members, and these roles interact indirectly through stigmergy. On the other hand, communication-based cooperation may or may not involve roles performed by its team members, but the team members are able to synchronize through direct communication with each other.

In the following section, these cooperative strategies will be characterized further by testing how robustly they evolve and how robustly the evolved agents perform under various extreme conditions.

\section{Robustness of Strategy Evolution and Performance}

Section 5 showed that when minimal neural-network architectures are used for each approach, the coevolutionary approach learns faster than the centralized approach, and the team without communication learns faster than the team with communication. It is important to verify that these results hold when equivalent neural-network architectures are used across all approaches. Also, since the evolved non-communicating strategies include fixed roles, it is necessary to demonstrate that they are robust against changes in the prey's behavior. It is also important to show that evolution can discover non-communicating strategies robustly even when the predators are initially placed randomly. Another interesting issue is whether communicating teams can be biased by design to learn role-based, rather than communication-based cooperative behavior. Finally, it is important to demonstrate that coevolution of heterogeneous agents is indeed necessary to solve the task. Each of these issues is studied in a separate experiment in this section.

\subsection{Do the results hold across equivalent network architectures?}

When the network architectures were optimized separately for each approach, the coevolutionary approach learned faster than the centralized approach (Section 5.2), and teams without communication faster than teams with communication (Section 5.3). However, it is unclear how much of this result is due to the different search-space sizes (i.e. different number of weights that need to be optimized), and how much is due to the centralized vs. distributed control strategy itself, or to the availability of communication.

In the centralized approach, the neural network had 9 hidden units and 21 connections per unit for a total of 189 weights (Figure 4), whereas the network used in the coevolutionary approach (with communication) had 8 hidden units and 11 connections per unit for a total of 88 weights (Figure 5); such a smaller search space may allow learning to progress significantly faster. The same issue arises in comparing communicating vs. non-communicating networks: The latter approach has 3 hidden units and 7 connections per unit for a total of 21 weights (Figure 6).

Of course, such small search spaces are possible precisely because the approaches are different, but it is still interesting to verify that the results are not completely due to search complexity. To this end, the experiments in Sections 5.2 and 5.3 were repeated with identical network architectures across all approaches. Specifically, all approaches use the architecture of the centralized approach, with 9 hidden units, 6 inputs, and 15 outputs. When some inputs are not part of the approach (such as teammate locations in the noncommunicating approach), random noise values are used for them; outputs that are not used are simply 


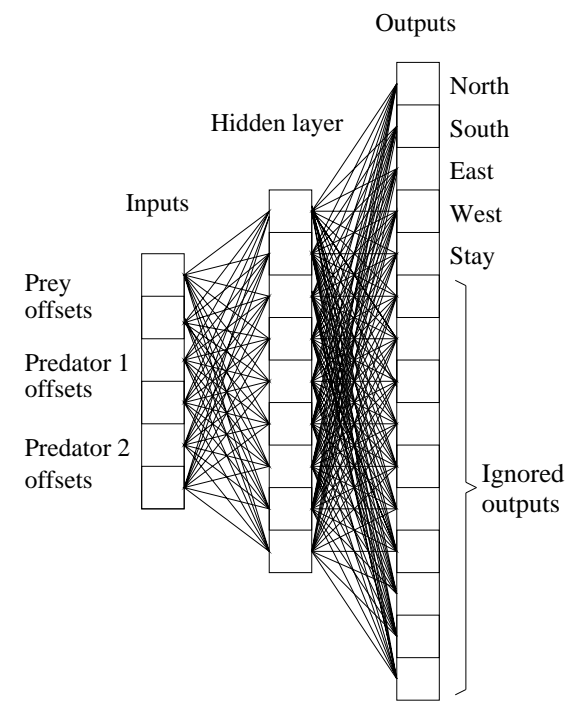

(a)

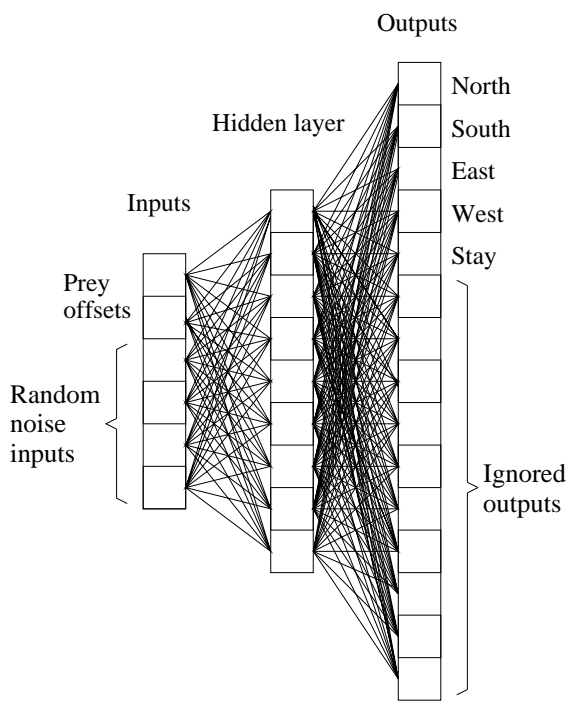

(b)

Figure 12: The large-network versions of the communicating and non-communicating controllers. (a) The network used in the autonomous communicating approach; ten of the outputs are ignored. (b) The network used in the autonomous non-communicating approach; ten of the outputs are ignored, and four inputs are fed random noise. In both cases, there are nine hidden units, and the chromosomes for each hidden layer unit consists of 21 real-valued numbers (six inputs and 15 outputs), which is the same as in the central-controller approach.

ignored (Figures $12 a, b$ ). The learning performance of the coevolutionary approaches with such large networks is compared against that of the original centralized approach, providing a comparison of the three different approaches given a uniform search space.

Figure 13 presents the results for these experiments. Using larger neural networks did not significantly change the number of required generations to learn the task for either of the coevolutionary approaches (with or without communication). As a result, the relative differences in learning time between the three approaches are preserved. Therefore, the better performance of the coevolutionary over the centralized approach, and that of the coevolutionary non-communicating over the coevolutionary communicating approach, are indeed due to the approaches themselves, and not simply a consequence of being able to search in a smaller space.

\subsection{Are the strategies robust against novel prey behavior?}

Although the non-communicating networks work together like a well-trained soccer team, soccer (like most interesting real-world tasks) is unpredictable. For example, a player from the other team may intercept a pass, in which case the team members will have to quickly adapt their strategy to cope with the new situation. To determine how the non-communicating team can deal with such unpredictability, three further experiments were conducted where the ten teams were pitted against a prey that behaved differently from those encountered during evolution. For comparison, the same tests were also run for the communicating teams. Since the non-communicating teams' predators act according to rigid roles, they might not be able to adapt as well as the communicating teams' more apparently flexible agents.

In the first experiment, the prey moved three times faster than usual (by moving three steps each time) and in a random direction $20 \%$ of the time; in the second test, three times faster than usual and in a random direction $50 \%$ of the time; and in the third, the prey always moved right. The results, summarized in 


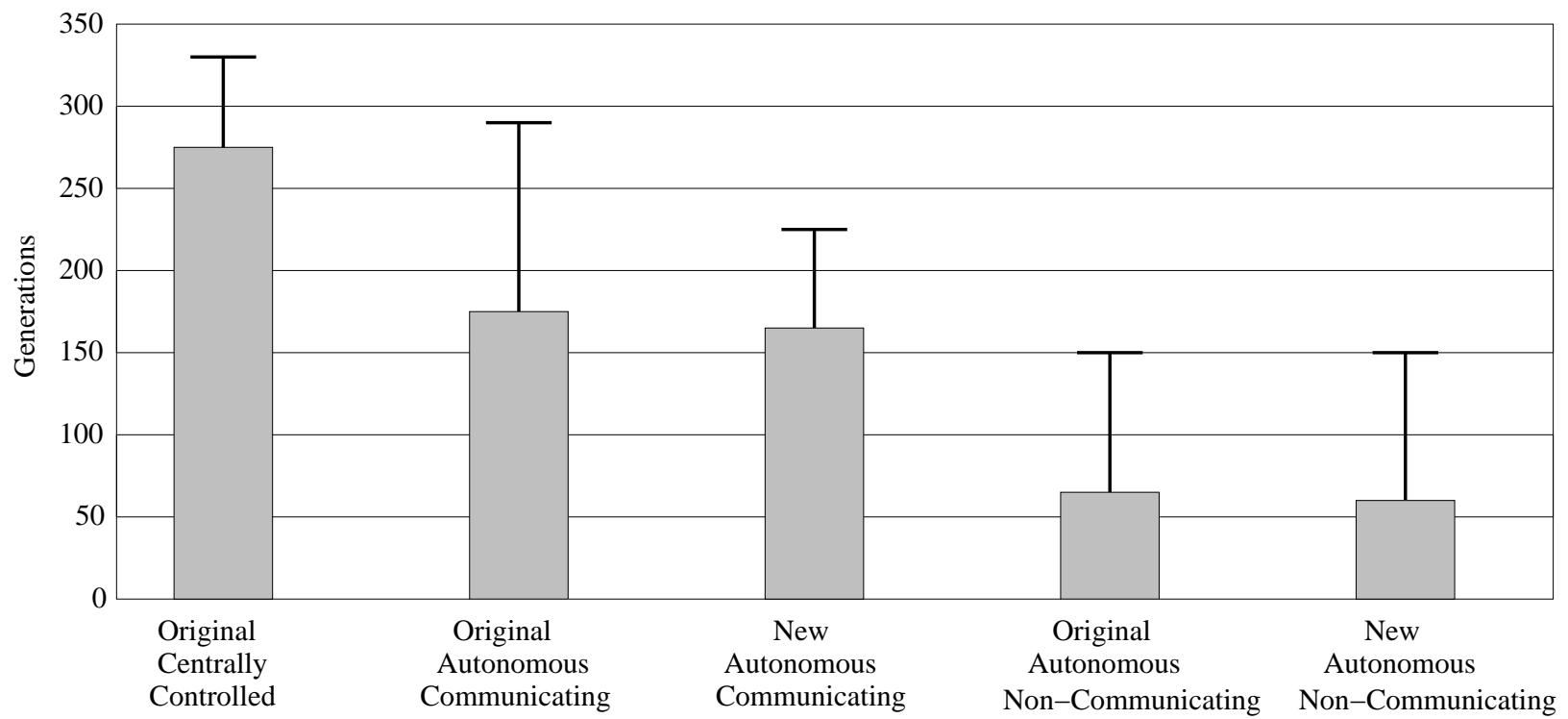

Figure 13: Learning performance for each approach with equivalent network architectures. The average number of generations, with standard deviation, required to solve the task is shown for each approach. The performance is about the same as with minimal architectures, and the relative performance between approaches is preserved. The differences between the approaches are statistically significant with $p>0.95$.

Figure 14, are surprising: The non-communicating teams are more robust against unpredictable preys than the communicating teams. Apparently, the first two prey behaviors, which are noisy versions of the original behavior, are still familiar enough so that the rigid roles are effective: The teams still catch the prey about $50 \%$ of the time. The agents only have to track the occasional erratic movement, otherwise their strategy is effective as is. The communicating teams, however, have a narrower range of adaptable situations, particularly because their agents tend to switch strategies and roles based on the current state of the world, and thus get easily confused by the unexpected prey actions. In the third case, where the prey always moves right, both teams are unable to adapt. This behavior is consistently novel, and the agents are evolved not to expect it.

In sum, teams that have delegated rigid and specific roles to their members may be more tolerant to noisy or unusual situations, as long as the basic strategy is still valid.

\subsection{Are the strategies robust with random deployment?}

In all experiments so far, the predators always started at the bottom left corner of the world, and only the prey's initial position was varied. Such a limitation makes it possible to analyze the resulting behaviors systematically. However, it is important to demonstrate that similar behavior evolves in the more general case when the predators' positions are initially random. The issue is, will the non-communicating networks be able to establish effective roles even when the predators' random initial positions make the initial state uncertain during learning?

The experiment was set up as described in Section 5.1, except that predators were placed randomly in the world during both evolution and benchmark tests. Furthermore, the number of evaluations per trial during evolution was increased from 9 to 45 , in order to obtain a sufficient sample of the different starting positions. The number of evaluations during benchmark tests was increased from 9 to 900 for the same reason (whereas relatively sparse sampling is sufficient to guide evolution, the benchmarks need to measure 


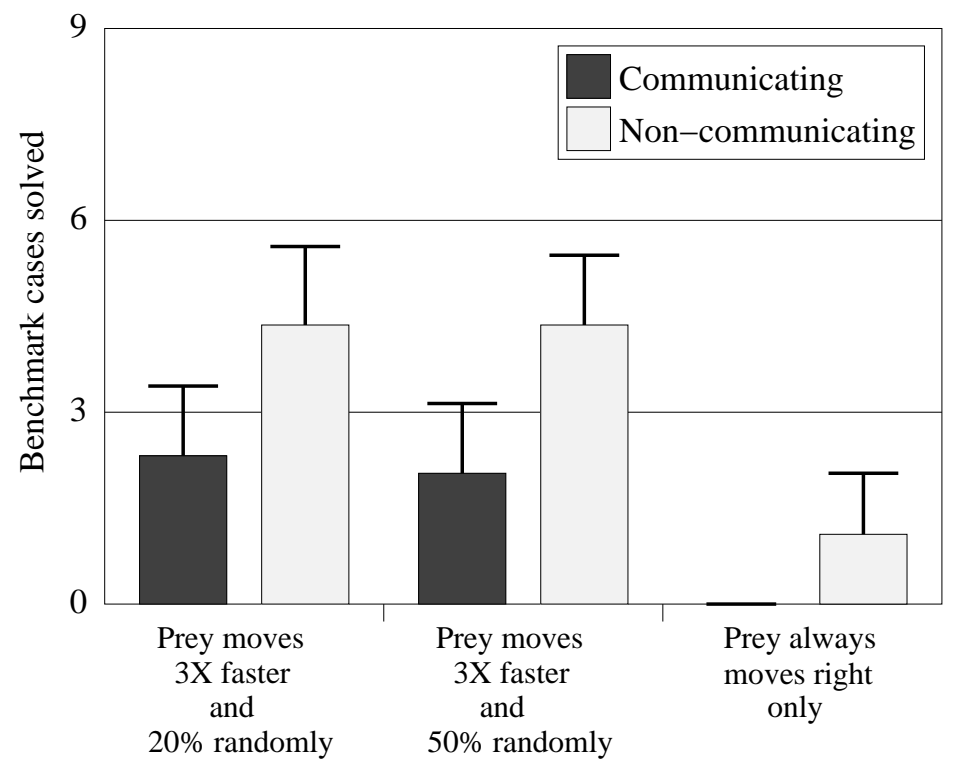

Figure 14: Robustness of communicating and non-communicating teams against novel prey behavior. In the first test, the prey moved three steps each time and in a random direction $20 \%$ of the time; in the second, three steps and randomly $50 \%$ of the time; and in the third, always right. The results were averaged over the ten runs of each approach. Surprisingly, the non-communicating teams performed significantly better than the communicating teams ( $p>0.99$ for all three tests), even though the communicating strategies were more flexible. The non-communicating teams tolerate the occasional novel behavior well as long as their basic strategy is valid; however, even they cannot cope if the prey employs a consistently different strategy.

performance more accurately). A network was deemed successful if it caught the prey in 750 of the 900 tests.

The minimum effective network sizes were determined as in Section 5.1. In this more challenging task, they were found to be 13 hidden units for the centrally controlled and communicating autonomous networks, and 5 hidden units for the non-communicating networks.

Figure 15 shows how long it took each approach to solve the task, averaged over 10 runs. Although this task was much harder, the conclusions are the same as before: The communicating autonomous teams were easier to evolve than central controllers, and the non-communicating networks easier than the communicating networks. The strategies learned were also similar to those with fixed initial placement of predators.

\subsection{Can evolution be biased toward role-based cooperation?}

As discussed in Section 6, the communicating networks could in principle evolve non-communicating behavior by learning zero weights on the connections from those input units that specify the other predators' positions. It does not happen, and the reason may be that it is difficult for evolution to turn off all such connections simultaneously; it is easier to instead discover a competent communicating solution, utilizing those inputs and weights as well.

An interesting question is whether evolution would discover role-based cooperation if it was biased to do so from the beginning. Such an experiment was run by setting all the communicating weights to zero in the initial population; the other weights were initialized randomly as usual. Evolution only had to keep the communicating weights at zero while developing the rest of the network to solve the task. As a comparison, networks with all weights initially zero were also evolved, and the results compared to normal evolution of 


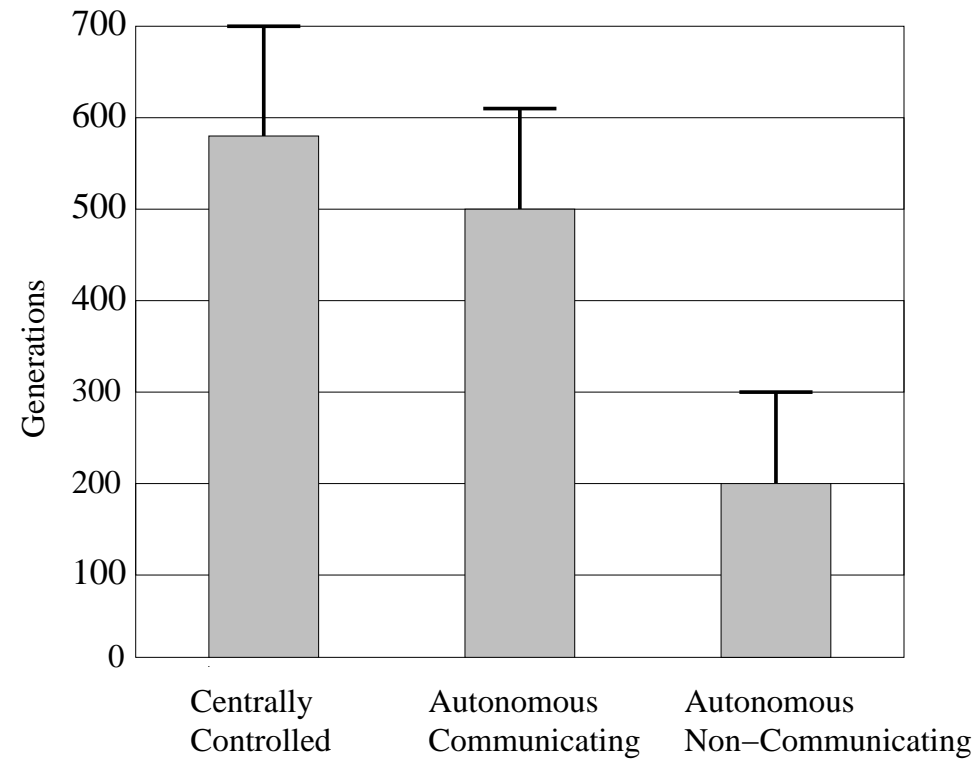

Figure 15: Learning performance of the three approaches when the predators start at random locations. In this harder task, evolution took longer than with fixed initial placement of predators in all cases. Moreover, as before, the non-communicating teams were significantly easier to evolve than communicating teams $(p>0.99)$, which were slightly easier than central controllers $(p>0.90)$. The team behaviors were also similar to those evolved earlier.

communicating networks.

The action dependencies that evolved are shown in Figure 16; the results were averaged over 10 runs. The results show that such an initial bias does have an effect: Evolution discovers behaviors where the actions depend significantly less often on the positions of other predators. With communication weights initially at zero, $14 \%$ of the actions depend on them, and with all weights initially zero, $48 \%$; in contrast, the earlier evolved networks with all randomly-initialized weights exhibit $91 \%$ dependency. Qualitatively the behaviors of the initially biased networks consist of mostly role-based cooperation, with occasional switches or deviations in the roles of the predators.

The performance in terms of number of generations needed to learn the task is shown in Figure 17. Teams with communication weights initialized to 0 show a significant improvement in evolution time: The number of generations required, compared to the normal communicating teams, dropped by a factor of three, making it as fast as the non-communicating teams. When all weights are initialized to 0 , the improvement in evolution time was found to be insignificant.

These results show that evolution can indeed discover role-based cooperation, and the number of generations required can be comparable to that needed by an architecture that forces role-based behavior (i.e. the non-communicating networks); however, the initial state needs to be biased the right way, and the role-based cooperation discovered may not be perfect. In other words, it is still important for the designer of a multiagent system to recognize whether role-based cooperation could work in the task, and utilize the appropriate architecture to evolve it.

\subsection{Is Coevolution Necessary?}

Although the performance of cooperative coevolution looks convincing, it does not necessarily mean that coevolution is essential for the task. Perhaps it is possible to evolve good predators individually, and just put 


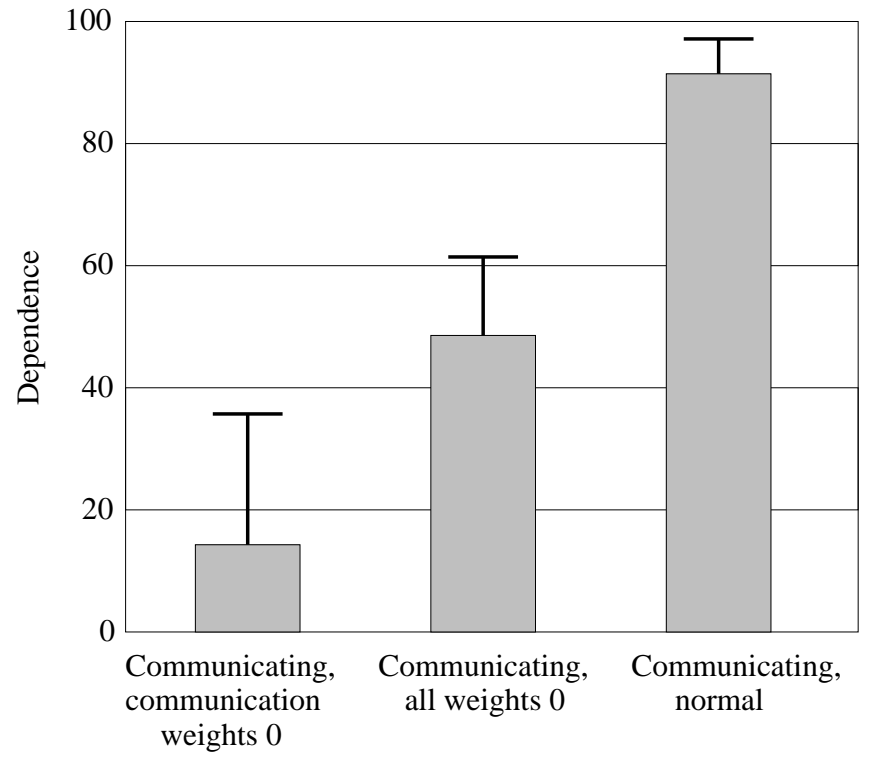

Figure 16: Degrees of dependence of communicating teams biased to evolve role-based cooperation. The communicating teams with communication weights initialized to 0 evolved behaviors that were $14 \%$ dependent on the positions of teammates; with all weights initialized to 0 , the evolved behaviors were $48 \%$ dependent. In both cases, the evolved behaviors were significantly less dependent on teammates compared to those of the normal (randomlyinitialized) communicating teams, which was $91 \%$ dependent on teammates (all differences are statistically significant with $p>0.99$ ).

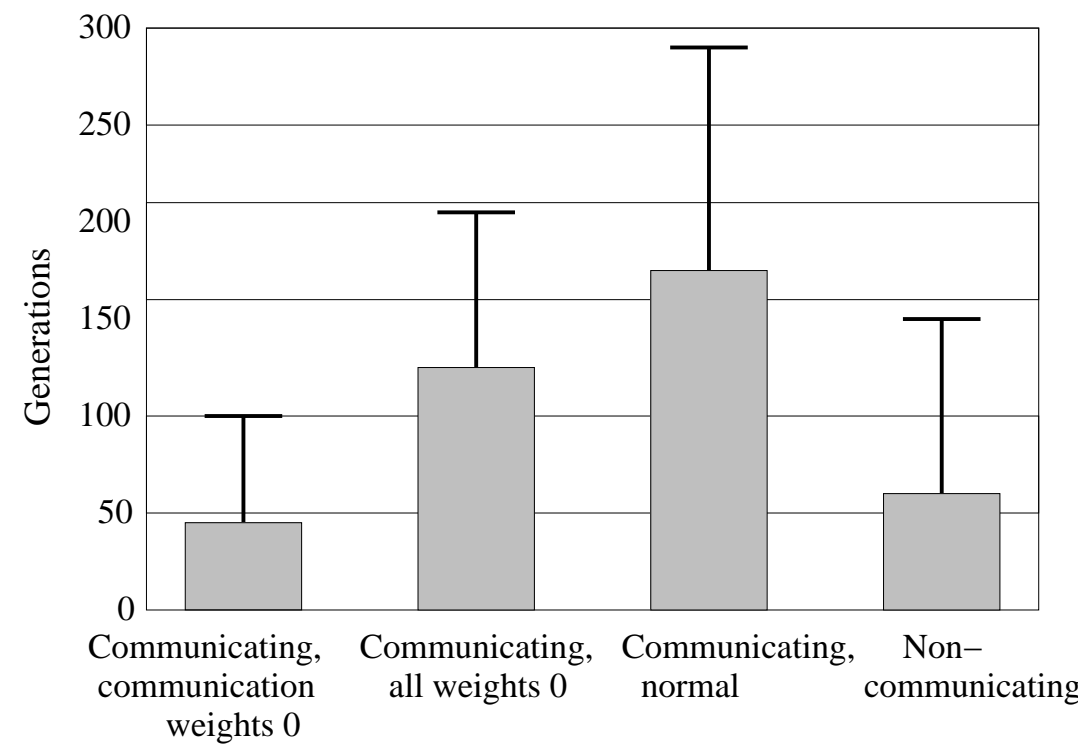

Figure 17: Learning performance of communicating teams biased to evolve role-based cooperation. The communicating teams with communication weights initialized to 0 learned the task significantly faster than the normal communicating teams $(p>0.99)$, taking about the same number of generations as the non-communicating teams $(p=0.68)$. On the other hand, with all weights initialized to 0 , learning was as slow as with normal communicating teams $(p=0.89)$, and significantly slower than with communicating weights initialized to 0 as well as with non-communicating teams $(p>0.90)$. 


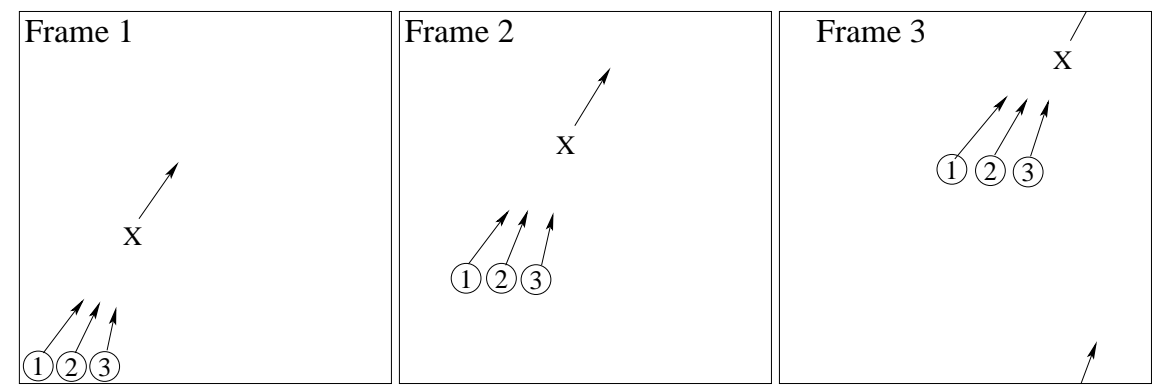

Figure 18: A strategy of three individually evolved predators placed on the same environment. The predators chase the prey together in the nearest direction but are unable to catch it.

them together into the domain? This subsection demonstrates experimentally that such an approach is not sufficient, and the agents indeed must be evolved together to solve the task.

A single predator without communication inputs (as shown in Figure 6) was evolved alone in the preycapture domain with incremental learning, using the standard ESP method as described in Section 4 and Figure 2. The predator was allowed to evolve until it could no longer improve its fitness. This process was repeated three times, each time with a new predator, to produce three independent but competent predators. These three predators were then put into the same environment and evaluated in the prey-capture task.

The results clearly support coevolution. When a predator evolves alone, it is never able to catch the prey, since the prey moves at the same speed as the predator. It learns to chase the prey but is never able to reduce the distance between them, and is only able to prevent the prey from increasing it. When the three individually evolved predators are put together against the prey, they all chase the prey in the nearest direction, and are unable to catch it at all-the prey keeps running and maintains the distance (Figure 18). In other words, coevolution is necessary in this task to evolve successful cooperative behavior.

\subsection{Are Heterogeneous Agents Necessary?}

Even though a team of individually evolved predators cannot catch the prey, it is possible that a homogeneous team, i.e. one where all predators are controlled by identical networks, could. Such a team can be successful only when the agents communicate; otherwise they would all employ the same behavior, and fail like the team in the previous section.

In a separate experiment, communicating networks were evolved through the standard ESP method. Each network was evaluated by making three copies of it, each controlling one of the three predators in a team; the fitness of such a homogeneous team was then taken as the network's fitness. Networks of different sizes were evolved, from 6 hidden units up to 16, with 10 runs at each size.

The success rate was extremely low: The homogeneous teams were able to solve the task only 3 times out of the 100 total runs. Apparently, it was difficult for evolution to discover a way to establish the roles initially in the beginning of the chase. In the heterogeneous communicating teams all agents are different and their behaviors diverge in the beginning, and the agents can immediately adopt a strategy that fits the situation. In the homogeneous team, all predators initially perform the same actions, and it is difficult to break the symmetry. A more effective form of communication might be to signal roles rather than simply sensing teammates' locations; each predator in turn would decide on a distinct role (such as chase northward, or block horizontally), and communicate that to its teammates. Alternatively, evolving heterogeneous networks allows symmetry breaking to occur naturally, resulting in effective heterogeneous behaviors without a negotiation phase. 


\section{Discussion}

In Section 5.2, a central controller was found to take over 50\% longer to evolve than autonomous cooperating controllers to solve the pursuit-evasion task. Cooperative coevolution is able to decompose the task into simpler roles, thereby making it easier to discover solutions.

Such decomposition is a special case of speciation in evolutionary systems. Speciation has been widely used to maintain diversity in evolution. Using techniques such as islands and fitness sharing [35, 28, 22], separated populations are encouraged to diverge, resulting in more efficient search of the solution space. If these separated populations are further evaluated jointly and rewarded with a global fitness, they tend to converge to heterogeneous policies that work well together. This is the driving mechanism behind cooperative coevolution and also behind ESP and its multi-agent extension. ESP preserves diversity across populations of neurons and networks, because these populations are disjoint by design. Although diversity is gradually lost within each population as evolution focuses on a solution, diversity is maintained between subpopulations, and diverse and complementary roles result. Thus, the observed cooperation between predators is a consequence of cooperation between populations during evolution.

Section 5.3 presented the result that predators without knowledge of teammates' relative locations evolve to cooperate and solve the task more than twice as fast than predators with such knowledge. This result is interesting because such knowledge from communication allows each predator to make decisions based directly on where the other predators are, as well as where the prey is; on the other hand, non-communicating predators do not have such knowledge, and have to learn to coordinate using stigmergy. At first glance, this result seems attributable to three factors. First, allowing communication usually requires more structure (the minimal communicating autonomous controllers have eight neurons with 11 weights each, while the minimal non-communicating autonomous controllers have three neurons with seven weights each), which translates to a larger search space. However, this difference turns out to be unimportant: As discussed in Section 7.1, when the same architecture is used across all approaches, the non-communicating approach still outperforms the communicating approach substantially.

The second potential factor is that communication presents more information for evolution to understand and organize, which might take more evolutionary generations. While it is theoretically possible for evolution to learn to discard the unnecessary information, Section 7.4 demonstrated that evolution instead attempts to make use of it. On the other hand, Section 7.1 showed that it takes evolution virtually no time to learn to discard random noise fed into the extra inputs of the non-communicating approach: The number of generations taken to solve the task is the same as without noisy inputs. The conclusion is that it is easy for evolution to discard noisy, random activations given as inputs, but difficult to discard inputs such as teammates' relative positions that "make sense," even if they are marginally useful at best.

The most important factor in explaining the better performance of the non-communicating over the communicating approach lies in the different behaviors learned in each approach, and their relationship to the powerful decompositional property within ESP itself. As was discussed in Section 6, the noncommunicating team always employed a single strategy where each agent had a specific role, whereas the communicating team tended to utilize variations and combinations of two or more strategies with roles that were not as well delineated. During evolution, each non-communicating subpopulation converges toward optimizing specific functions such that, as long as each agent performs its role right, the team solves the task successfully, even though the team members are independent of one another. Evolution without communication thus places strong pressure on each predator to perform its assigned role well. These roles are assigned through simultaneous adaptive niching: As one agent begins to converge to a particular behavior, the other agents that behave complementarily are rewarded, and themselves begin to niche into such roles; this adaptation in turn yields a higher fitness, and all predators begin to converge into cooperative roles. In 
this way, a stigmergic form of coordination between team members is fast to emerge.

Franklin [8] pointed out that communication abilities involve non-trivial costs, and suggested that in some cases coordination without communication may be an advantage over communication-based coordination. Our findings suggest that this is indeed true: First, communication presents extra information that ESP takes time to learn to organize and utilize, even if such information is not crucial in solving the task; second, communication raises the structural requirements of the communicating controller in terms of number of hidden units and weights. Even if this does not cause an increase in the number of generations to solve the task, it increases the computational resources needed to store, evaluate and operate upon the larger structures. On the other hand, stigmergy is simpler and faster for ESP to learn due to the powerful decompositional properties of the algorithm, and is utilized more beneficially in the task to find a rigid, efficient solution.

In general, the results in this paper suggest that it is better to use the most parsimonious architecture possible to solve a task, rather than an architecture with full capabilities and the hope that evolution will guide learning to discard the unnecessary capabilities. First, it is usually faster to evolve simpler architectures. Efficiency becomes important especially with difficult real-world problems, which tend to be noisy and unpredictable. For example, Section 7.3 showed that when predators start in random initial positions, the number of generations required to learn the task increased significantly, and each generation took longer because more sampling was required. Furthermore, Section 7.4 demonstrated that evolution may not learn to discard extraneous capabilities, but rather learns to use them in less efficient and less robust solutions. In domains that are completely role-based, a particularly efficient way to simplify a system's architecture is to discard communication between agents; the system will then learn the task faster, and learn more efficient and robust solutions.

Of course, not all multi-agent domains may be as efficiently solved through role-based cooperation. For example in the prey-capture domain where a capture configuration is necessary (as used by Benda [6] and Haynes and Sen $[16,15])$ it would be very difficult for the agents to guess the exact locations of the other agents to achieve successful capture. In contrast, if the agents can let other agents know where they are, they can coordinate their positions. Just as team behaviors were classified as either role-based or communicationbased in Section 6.2, it may be useful to classify multi-agent domains likewise: Role-based cooperative domains can be solved more efficiently by teams employing role-based cooperation without direct communication, while communication-based cooperative domains require some degree of direct communication between teammates to be solved efficiently. The prey-capture domain in this paper is role-based, in that direct communication is not necessary at all.

Given a multi-agent problem, it is then extremely useful to identify it as a communication-based or role-based domain (or equivalently, determine whether direct communication between teammates is useful or not), so that one can choose an appropriate learning approach for it. As discussed above, employing a communicating team in a role-based domain may not give the most efficient or most robust solution, while a non-communicating team may not even be able to solve a task that is communication-based. While it may not be possible to identify the type of domain in every case, there are a few observations that are likely to be helpful. First, in a task that requires temporal synchronization, communication between teammates is likely to be essential, and the domain is therefore communication based. An example is a prey-capture domain where all predators have to move to occupy a capture configuration simultaneously. Second, in a task where essential, non-redundant roles may not always complete, communication is likely to be required so that agents can seek assistance form teammates, or to notify them that a subtask cannot be completed. For example, if the prey-capture domain included irregularly-placed obstacles, stigmergic coordination might be insufficient to solve the task. On the other hand, there are likely to be several real-world domains where rolebased cooperation is effective. For example, in controlling a bank of elevators in a building, each agent's 
role could be to serve a specific range of floors; in coordinating agents to search the web for information, each agent could cover a separate web region. In such domains, communication may turn out to be a source of noise that diverts teams from the best solution. Systematically identifying such tasks and applying rolebased cooperative coevolution to them constitutes a most interesting direction for future work.

\section{Conclusion}

The experiments reported in this paper show that coevolving several autonomous, cooperating neural networks to control a team of agents can be more efficient and robust than evolving a single centralized controller, and that Multi-Agent ESP is an efficient and natural method for implementing such multi-agent cooperative coevolution. Furthermore, a class of domains was identified, called role-based cooperative domains, where direct communication is not necessary for success, and may actually make evolution less effective. Instead, a team of non-communicating agents can be evolved to utilize stigmergic coordination to solve the task more efficiently and robustly. Many real world tasks appear to fall in this category of domains. Recognizing such tasks and applying the cooperative coevolution approach to them, as well as studying the limits of stigmergic coordination in dealing with novel and changing environments, are the main directions of future work in this area.

\section{Acknowledgments}

This research was supported in part by the National Science Foundation under grants IIS-0083776 and EIS-0303609 and the Texas Higher Education Coordinating Board under grant ARP-003658-476-2001. We thank Bobby Bryant for insightful suggestions on how to test cooperation and communication, and Tino Gomez for suggestions on adapting ESP to multi-agent systems, as well as the anonymous reviewers for suggestions on stigmergy and network size experiments.

\section{References}

[1] Anderson, C. W. (1989). Learning to control an inverted pendulum using neural networks. IEEE Control Systems Magazine, 9:31-37.

[2] Balch, T. (1997). Learning roles: Behavioral diversity in robot teams. In Sen, S., editor, Multiagent Learning: Papers from the AAAI Workshop. Technical Report WS-97-03, 7-12. Menlo Park, CA: American Association for Artificial Intelligence.

[3] Baldassarre, G., Nolfi, S., and Parisi, D. (2003). Evolving mobile robots able to display collective behaviors. Artificial Life, 9:255-267.

[4] Barto, A. G., Sutton, R. S., and Anderson, C. W. (1983). Neuronlike adaptive elements that can solve difficult learning control problems. IEEE Transactions on Systems, Man, and Cybernetics, SMC13:834-846.

[5] Batali, J. (1994). Innate biases and critical periods: Combining evolution and learning in the acquisition of syntax. In Brooks, R. A., and Maes, P., editors, Proceedings of the Fourth International Workshop on the Synthesis and Simulation of Living Systems (Artificial Life IV), 160-171. Cambridge, MA: MIT Press. 
[6] Benda, M., Jagannathan, V., and Dodhiawala, R. (1986). On optimal cooperation of knowledge sources-An empirical investigation. Technical Report BCS-G2010-28, Boeing Advanced Technology Center.

[7] Elman, J. L. (1991). Incremental learning, or The importance of starting small. In Hammond, K. J., and Gentner, D., editors, Proceedings of the 13th Annual Conference of the Cognitive Science Society, 443-448. Hillsdale, NJ: Erlbaum.

[8] Franklin, S. (2001). Coordination without communication. Technical report, Institute for Intelligent Systems, Department of Mathematical Sciences, University of Memphis.

[9] Gomez, F. (2003). Robust Non-Linear Control through Neuroevolution. PhD thesis, Department of Computer Sciences, The University of Texas at Austin.

[10] Gomez, F., and Miikkulainen, R. (1997). Incremental evolution of complex general behavior. Adaptive Behavior, 5:317-342.

[11] Gomez, F., and Miikkulainen, R. (1999). Solving non-Markovian control tasks with neuroevolution. In Proceedings of the 16th International Joint Conference on Artificial Intelligence, 1356-1361. San Francisco: Kaufmann.

[12] Grassé, P.-P. (1959). La reconstruction du nid et les coordinations inter-individuelles chez Bellicositermes natalensis et Cubitermes sp. la théorie de la stigmergie: Essai d'interpretation des termites constructeurs. Insectes Sociaux, 6:41-83.

[13] Gruau, F., Whitley, D., and Pyeatt, L. (1996). A comparison between cellular encoding and direct encoding for genetic neural networks. In Koza, J. R., Goldberg, D. E., Fogel, D. B., and Riolo, R. L., editors, Genetic Programming 1996: Proceedings of the First Annual Conference, 81-89. Cambridge, MA: MIT Press.

[14] Haynes, T., and Sen, S. (1996). Evolving behavioral strategies in predators and prey. In Weib, G., and Sen, S., editors, Adaptation and Learning in Multi-Agent Systems, Lecture Notes in Computer Science, 113-126. Berlin: Springer.

[15] Haynes, T., and Sen, S. (1997). Crossover operators for evolving a team. In Koza, J. R., Deb, K., Dorigo, M., Fogel, D. B., Garzon, M., Iba, H., and Riolo, R. L., editors, Genetic Programming 1997: Proceedings of the Second Annual Conference, 162-167. San Francisco: Kaufmann.

[16] Haynes, T. D., and Sen, S. (1997). Co-adaptation in a team. International Journal of Computational Intelligence and Organizations, 1:231-233.

[17] Holland, J. H. (1986). Escaping brittleness: The possibilities of general-purpose learning algorithms applied to parallel rule-based systems. In Michalski, R. S., Carbonell, J. G., and Mitchell, T. M., editors, Machine Learning: An Artificial Intelligence Approach, vol. 2, 275-304. San Francisco: Kaufmann.

[18] Holland, O., and Melhuish, C. (1999). Stigmergy, self-organization, and sorting in collective robotics. Artificial Life, 5:173-202.

[19] Jim, K.-C., and Giles, C. L. (2000). Talking helps: Evolving communicating agents for the predatorprey pursuit problem. Artificial Life, 6(3):237-254. 
[20] le Cun, Y., Denker, J. S., and Solla, S. A. (1990). Optimal brain damage. In Touretzky, D. S., editor, Advances in Neural Information Processing Systems 2, 598-605. San Francisco: Kaufmann.

[21] Luke, S. (1998). Genetic programming produced competitive soccer softbot teams for Robocup97. In Koza, J. R., Banzhaf, W., Chellapilla, K., Deb, K., Dorigo, M., Fogel, D. B., Garzon, M. H., Goldberg, D. E., Iba, H., and Riolo, R., editors, Genetic Programming 1998: Proceedings of the Third Annual Conference, 214-222. Kaufmann.

[22] Mahfoud, S. W. (1995). Niching Methods for Genetic Algorithms. PhD thesis, University of Illinois at Urbana-Champaign, Urbana, IL.

[23] Meuleau, N., Peshkin, L., Kim, K.-E., and Kaelbling, L. P. (1999). Learning finite-state controllers for partially observable environments. In Proceedings of the Fifteenth International Conference on Uncertainty in Artificial Intelligence, 427-436. San Francisco: Kaufmann.

[24] Miller, G., and Cliff, D. (1994). Co-evolution of pursuit and evasion I: Biological and game-theoretic foundations. Technical Report CSRP311, School of Cognitive and Computing Sciences, University of Sussex, Brighton, UK.

[25] Moriarty, D. E. (1997). Symbiotic Evolution of Neural Networks in Sequential Decision Tasks. PhD thesis, Department of Computer Sciences, The University of Texas at Austin. Technical Report UTAI97-257.

[26] Moriarty, D. E., and Miikkulainen, R. (1996). Efficient reinforcement learning through symbiotic evolution. Machine Learning, 22:11-32.

[27] Moriarty, D. E., and Miikkulainen, R. (1997). Forming neural networks through efficient and adaptive co-evolution. Evolutionary Computation, 5:373-399.

[28] Muhlenbein, H., Schomisch, M., and Born, J. (1991). The parallel genetic algorithm as a function optimizer. In Belew, R. K., and Booker, L. B., editors, Proceedings of the Fourth International Conference on Genetic Algorithms, 271-278. San Francisco: Kaufmann.

[29] Paolo, E. D. (2000). Behavioral coordination, structural congruence and entrainment in a simulation of acoustically coupled agents. Adaptive Behavior, 8:25-46.

[30] Pendrith, M. (1994). On reinforcement learning of control actions in noisy and non-Markovian domains. Technical Report UNSW-CSE-TR-9410, School of Computer Science and Engineering, The University of New South Wales, Sydney, Australia.

[31] Potter, M. A., and Jong, K. A. D. (2000). Cooperative coevolution: An architecture for evolving coadapted subcomponents. Evolutionary Computation, 8:1-29.

[32] Quinn, M., Smith, L., Mayley, G., and Husbands, P. (2002). Evolving teamwork and role-allocation with real robots. In Proceedings of the Eighth International Conference on Artificial Life, 302-311. Cambridge, MA: MIT Press.

[33] Ramachandran, S., and Pratt, L. Y. (1992). Information measure based skeletonisation. In Moody, J. E., Hanson, S. J., and Lippmann, R. P., editors, Advances in Neural Information Processing Systems 4, 1080-1087. San Francisco: Kaufmann.

[34] Savage, T. (1998). Shaping: The link between rats and robots. Connection Science, 10:321-340. 
[35] Smith, R. E., Forrest, S., and Perelson, A. S. (1993). Searching for diverse, cooperative populations with genetic algorithms. Evolutionary Computation, 1:127-149.

[36] Smith, S. F. (1980). A Learning System Based on Genetic Adaptive Algorithms. PhD thesis, Department of Computer Science, University of Pittsburgh.

[37] Steels, L. (1996). Self-organising vocabularies. In Langton, C. G., and Shimohara, K., editors, Proceedings of the 5th International Workshop on Artificial Life: Synthesis and Simulation of Living Systems (ALIFE-96), 179-184. Cambridge, MA: MIT Press.

[38] Urzelai, J., Floreano, D., Dorigo, M., and Colombetti, M. (1998). Incremental robot shaping. Connection Science, 10:341-360.

[39] Wagner, K. (2000). Cooperative strategies and the evolution of communication. Artificial Life, 6:149179.

[40] Watkins, C. J. C. H., and Dayan, P. (1992). Q-learning. Machine Learning, 8(3):279-292.

[41] Werner, G. M., and Dyer, M. G. (1991). Evolution of communication in artificial organisms. In Langton, C. G., Taylor, C., Farmer, J. D., and Rasmussen, S., editors, Proceedings of the Workshop on Artificial Life (ALIFE '90), 659-687. Reading, MA: Addison-Wesley.

[42] Whitley, D., Dominic, S., Das, R., and Anderson, C. W. (1993). Genetic reinforcement learning for neurocontrol problems. Machine Learning, 13:259-284.

[43] Whitley, D., Gruau, F., and Pyeatt, L. (1995). Cellular encoding applied to neurocontrol. In Eshelman, L. J., editor, Proceedings of the Sixth International Conference on Genetic Algorithms, 460-469. San Francisco: Kaufmann.

[44] Whitley, D., Mathias, K., and Fitzhorn, P. (1991). Delta-Coding: An iterative search strategy for genetic algorithms. In Belew, R. K., and Booker, L. B., editors, Proceedings of the Fourth International Conference on Genetic Algorithms, 77-84. San Francisco: Kaufmann. 